SCIENTIFIC REPORT

\title{
Gorlin syndrome: the PTCH gene links ocular developmental defects and tumour formation
}

\author{
N K Ragge, A Salt, J R O Collin, A Michalski, P A Farndon
}

Br J Ophthalmol 2005;89:988-991. doi: 10.1136/bjo.2004.061390

Aim: To identify a gene linking microphthalmia with cyst with early onset medulloblastoma.

Methods: Mutation analysis of the PTCH gene.

Results: A mutation in exon 10 of the PTCH gene was identified, confirming a diagnosis of Gorlin syndrome.

Conclusions: This is the first genetically identified mutation giving rise to microphthalmia with cyst and provides a valuable link in the eye developmental gene pathway.

M icrophthalmia with cyst is a relatively rare ocular developmental defect that is caused by failure of optic fissure closure, thus forming part of the spectrum of colobomatous abnormalities. ${ }^{1-3}$ Although microphthalmia with cyst is usually an isolated phenomenon, it can occur in association with systemic abnormalities notably clefting syndromes, oculo-cerebro-cutaneous syndrome, branchiooculo-facial syndrome, and cardiovascular anomalies including coarctation of the aorta and atrial septal defects. ${ }^{4}$ However, any described conditions and genes (for example SHH (sonic hedgehog), or SIX3) associated with coloboma formation could in theory also be associated with microphthalmia or anophthalmia with cyst formation. ${ }^{5}$ Isolated microphthalmia with cyst is usually sporadic, with occasional familial occurrence described, ${ }^{7}$ including a family with autosomal recessive inheritance. ${ }^{8}$ All inheritance patterns have been described for microphthalmia with coloboma, and in theory this could also be seen in microphthalmia with cyst formation. ${ }^{1} 910$

Microphthalmia, with or without coloboma, is one of around 100 potential features seen in the naevoid basal cell carcinoma (BCC) syndrome or Gorlin syndrome, an autosomal dominant condition first described by Gorlin and Goltz. ${ }^{11} 12$ The other ocular features include anterior segment dysgenesis with cataract and Peter's anomaly, and vitreoretinal anomalies including epiretinal membrane formation, myelinated nerve fibres, and persistent fetal hyaloid anomalies. ${ }^{13}$ The main systemic features include developmental defects, such as skeletal abnormalities (bifid ribs and kyphoscoliosis), palmar and plantar pits, odontogenic keratocysts, falx calcification, as well as a predisposition to tumour development, including multiple basal cell carcinomas, medulloblastoma, and rhabdomyosarcoma. The gene for Gorlin syndrome has been identified as the human homologue of the Drosophila segment polarity gene, "patched,"14 known as the PTCH gene. We present an unusual case of microphthalmia with cyst associated with early medulloblastoma formation where a mutation within the PTCH gene has been identified, confirming a diagnosis of Gorlin syndrome. We discuss the implications of developmental ocular gene defects as indicators of tumour suppressor gene syndromes.
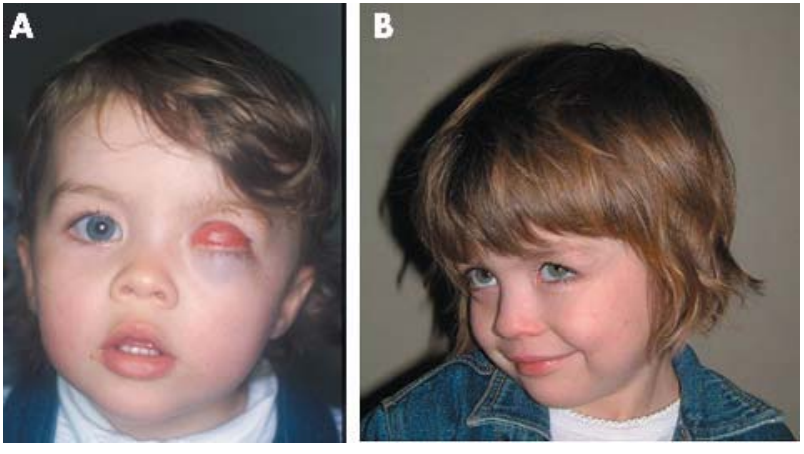

Figure 1 Facial appearance of patient with left microphthalmia and cyst showing appearance (A) before removal and (B) after removal of left orbital cyst with ocular prosthesis in place. (Photographs reproduced with the consent of the patient's parents.)

\section{CASE REPORT}

An asymptomatic girl with left microphthalmia and orbital cyst (fig 1A), was found to have a posterior fossa medulloblastoma on routine magnetic resonance imaging during preoperative assessment before planned excision of the cyst at the age of 2 years 4 months. She was born by normal vaginal delivery at 40 weeks gestation with a birth weight of $3.2 \mathrm{~kg}$ following an uncomplicated pregnancy. A large left orbital cyst was noted at birth, associated with a microphthalmic eye. The other eye was normal. Her early history was otherwise unremarkable.

At the time of the diagnosis of medulloblastoma she had healthy parents and a sibling aged 3 months and there was no other family history of malignancy or ocular problems. The magnetic resonance image (MRI) of the brain (fig 2) confirmed the cystic abnormality associated with the abnormal left eye and ruled out intracranial extension. However, it also demonstrated an enhancing posterior fossa mass consistent with a medulloblastoma that was distorting the cavity of the fourth ventricle and the cerebellar vermis. The MRI also showed a heterogeneously enhancing partly cystic pineal mass, the nature of which was unclear. The spine was clear.

At posterior fossa craniotomy a well circumscribed firm lesion was seen to the left of the midline anterior to the inferior cerebellar vermis. The lesion was removed in its entirety. Macroscopic pathological examination revealed a cystic nodule measuring $2 \mathrm{~cm}$ in diameter. On microscopy cerebellar folia and large areas of compact collagenous tissue were seen in which there were lymphocytes and scattered large aggregations of tumour cells. A dense network of collagen fibres percolated between the tumour cells. Mitoses and apoptotic bodies were present but there was no necrosis or vascular proliferation. The tumour cells expressed a

Abbreviations: $\mathrm{BCC}$, basal cell carcinoma; $\mathrm{SHH}$, sonic hedgehog 


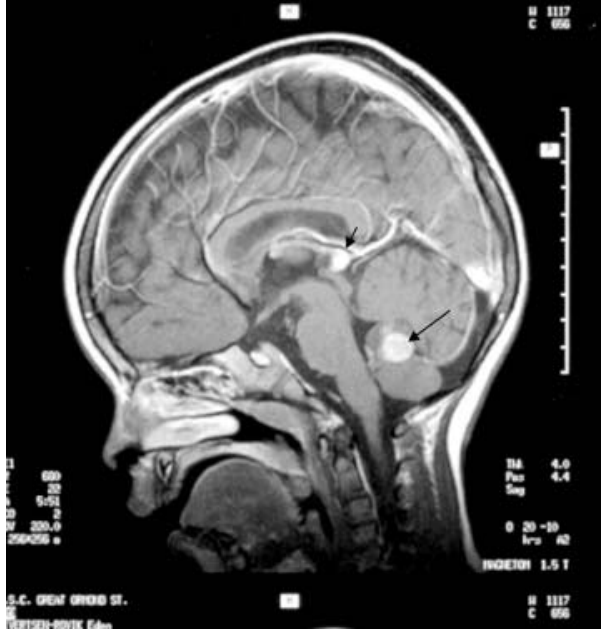

Figure 2 Sagittal section of MRI scan of patient demonstrating enhancing mass in the posterior fossa (long arrow), and a further enhancing lesion in the pineal region (short arrow).

number of neuroectodermal markers (MB84, CD 56, NSE), and the proliferation marker, Ki67, showed a high cell turnover in the cellular areas. In summary the features were consistent with a desmoplastic medulloblastoma.

After surgery, MRI of the brain also showed the previously noted heterogeneously enhancing partly cystic pineal mass but no evidence of residual posterior fossa tumour. Cerebrospinal fluid cytology did not reveal the presence of malignant cells. A Hickman line was inserted for delivery of treatment and bone marrow was harvested. Treatment was with the Infant PNET (primitive neuroectodermal tumour) protocol, which consists of six courses of dose intensive "induction" chemotherapy with growth factor support. This is usually followed by focal radiotherapy and consolidation chemotherapy. Following induction chemotherapy there was no evidence of residual or recurrent tumour. The parents decided against radiotherapy after careful discussion with the treating team and with a second oncologist, because of the possible neuropsychological late effects. Follow up included 3 monthly MRI spine and brain scans. The last was 30 months after surgery with no recurrence demonstrated. She had the orbital cyst excised at the age of 4 years 8 months.

The unusual association of early onset medulloblastoma with microphthalmia suggested this may be Gorlin syndrome and we undertook mutational analysis of the PTCH gene.

\section{Methodology}

Exons of the PTCH gene were screened by combined SSCP and heteroduplex analysis. ${ }^{15}$ Exons that showed a variant band pattern were sequenced to confirm the presence of a mutation.

\section{RESULTS}

A deletion of $\mathrm{G}$ at nucleotide position 1402 within exon 10 of the PTCH gene was detected by sequencing. This frameshift mutation results in the introduction of a stop codon within exon 10 and is predicted to result in the translation of a truncated PTCH protein (fig 3). Neither parent had this mutation on sequencing of lymphocyte DNA.

\section{DISCUSSION}

Although ocular phenotypes listed for Gorlin syndrome include microphthalmia and coloboma, these are relatively rare signs and no case of microphthalmia with cyst has been
A

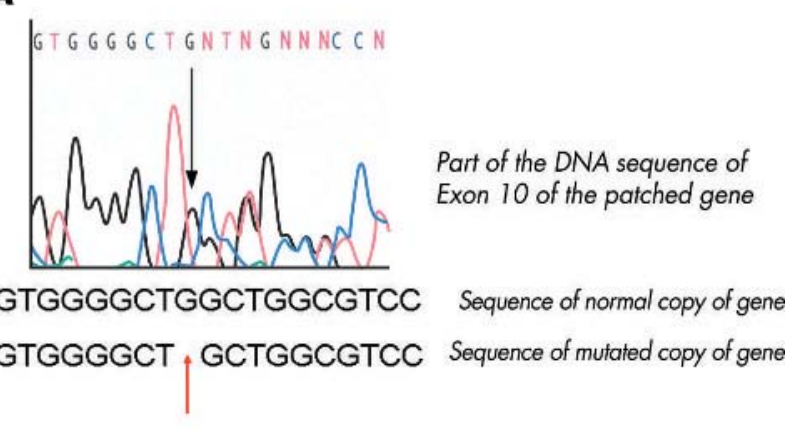

The "G" normally present at

nucleotide position 1402

has been deleted

B

\section{Predicted effect on patched protein}

Normal sequence

CTG GCT GGC GTC CTG CTG GTT ... TGC TCA TTG

Mutated sequence

CTG CTG GCG TCC TGC TGG TTG ... GCT CAT TGA

Deletion of $\mathbf{G}$ alters subsequent coding sequence STOP

A premature STOP message is generated. This truncates the protein made from that copy of the gene

C

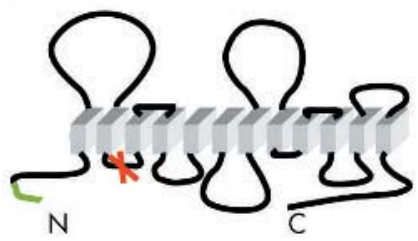

The mutation is predicted to truncate the protein where shown with complete loss of function

As one copy of the gene is still functioning normally, it would be expected that the patient has half the level of normally functioning patched protein

Figure 3 (A) DNA sequencing of patched gene in our patient shows deletion of " $G$ " at nucleotide position 1402. (B) Predicted effect on the patched protein. Deletion of $G$ causes a frameshift and subsequent stop codon which prematurely truncates the protein made from that copy of the gene. (C) Predicted effect of mutation on patched protein. The normal patched protein consists of 12 transmembrane domains and two large hydrophilic extracellular loops. The transmembrane domains show 100\% homology between mouse and human. The mutation is predicted to truncate the protein at the position marked with a cross leading to complete loss of function of that copy, and therefore haploinsufficiency.

previously reported. It is now well established that microphthalmia with cyst is a developmental defect caused by failure of the optic fissure to close and forms part of the coloboma spectrum of ocular developmental anomalies. ${ }^{1-3}$ The identification of a mutation within the PTCH gene, the gene known to give rise to Gorlin syndrome, highlights the importance of this gene in the ocular development gene pathway, particularly in the successful closure of the fetal fissure. 
The eye develops by a precisely orchestrated sequence of interdependent morphogenetic programmes allowing inductive interactions between tissues of different embryonic origin. All these ocular developmental processes are controlled by a complex network of regulatory genes which are co-expressed in precise temporal and spatial patterns to form various eye compartments. These genes are highly conserved across species. These same developmental genes are also expressed in other parts of the developing embryo and are important in organogenesis of other systems. Such observations provide an explanation for the plethora of associated phenotypes seen in cases of anophthalmia-microphthalmia.

The gene $P T C H$ is an important developmental regulator and tumour suppressor gene. The PTCH protein is a binding protein for SHH-which is crucial to many aspects of developmental patterning in the vertebrate embryo. PTCH functions with $\mathrm{SHH}$ as part of a dosage sensitive pathway resulting in activation of downstream target genes, including Smoothened and GLI. ${ }^{16}$ During central nervous system development, $\mathrm{SHH}$ is required for ventral specification along the entire neural axis. Heterozygous mutations within the SHH gene in humans can lead to holoprosencephaly, cyclops, and microphthalmia with coloboma. ${ }^{56}{ }^{17-19}$ Mice homozygous for mutations in the Ptch gene die as embryos and have massive neural tube defects. ${ }^{20}$ Heterozygote Ptch+/- mice are larger than wild type mice, and have hindlimb defects (for example, polydactyly or syndactyly), medulloblastomas, and soft tissue tumours. ${ }^{21}$ Of interest, mutations in Ptch in mice also cause vitreoretinal abnormalities similar to those seen in the human. ${ }^{13}$

The PTCH gene is an especially interesting gene since it acts both as a developmental gene and as a tumour suppressor gene akin to the retinoblastoma gene. ${ }^{22}$ The congenital anomalies are associated with a mutation in one copy of the PTCH gene and are predicted to be caused by gene dosage alterations in the hedgehog pathway during development. Our patient had a truncating PTCH mutation in one allele reducing the predicted amount of PTCH protein by $50 \%$. As her structural eye malformation was unilateral (the most usual pattern in Gorlin syndrome ${ }^{13}$ ) PTCH haploinsufficiency must interact with other localised effects such as the other genes expressed in the immediate vicinity, critical timing periods, and external factors. Loss of function of the second PTCH allele leads to the formation of jaw cysts, ${ }^{23}$ basal cell carcinomas, ${ }^{24}$ and in medulloblastoma, ${ }^{25}{ }^{26}$ as presumably in this case.

The identification of the mutation within the PTCH gene, and therefore a firm diagnosis of Gorlin syndrome, is important for several reasons. Firstly, it provides an explanation to the family for an apparently rare coincidence of a serious ocular developmental defect and early onset potentially life threatening medulloblastoma. Secondly, an understanding of the more benign natural history of early medulloblastoma in Gorlin syndrome provides reassurance to the family and clinicians and supports the serendipitous decision to withhold radiotherapy because of the risk of developing hundreds of BCCs in the treatment zone.. ${ }^{27}$ Indeed, in future, analysis of the gene expression patterns within medulloblastomas may assist in predicting the likely response to various chemotherapeutic agents. ${ }^{26}$ Thirdly, a knowledge of other potential features of Gorlin syndrome can lead to appropriate screening-for example, for odontogenic cysts, and advice about sun avoidance and use of sunscreen that may result in a reduction in the development of BCCs. Finally, genetic testing can be offered to the family to identify other members at risk so that surveillance for complications can be instituted.

Although often helpful in confirming the diagnosis, ${ }^{29}$ skull, chest, and spine radiographs were not undertaken in our patient's case because a pathogenic mutation had been identified on DNA analysis. Her continuing management for Gorlin syndrome ${ }^{30}$ will include orthopantograms for jaw cysts from about 8 years of age and detailed skin examination from puberty for BCCs.

As result of this study further work is in progress to screen other patients with microphthalmia-anophthalmia and other clinical abnormalities-for example, cleft lip-palate, midline abnormalities to establish the prevalence of PTCH mutations, which may not result in full Gorlin syndrome. We would like to draw attention to the association between developmental eye anomalies and tumour suppressor genes syndromes and to alert clinicians to potential future tumour development in those patients with apparently isolated developmental eye anomalies.

\section{ACKNOWLEDGEMENTS}

We would like to thank the family for their participation in our study and West Midlands Regional Genetics Laboratory, Birmingham Women's Hospital, Edgbaston, Birmingham for performing the molecular analysis. Nicola Ragge is a senior surgical scientist supported by the Academy of Medical Sciences/The Health Foundation.

\section{Authors' affiliations}

N K Ragge, A Salt, J R O Collin, Adnexal Service, Moorfields Eye Hospital, London, UK

N K Ragge, Department of Human Anatomy and Genetics, University of Oxford, Oxford, and Department of Ophthalmology, Birmingham Children's Hospital, Birmingham, UK

A Salt, A Michalski, Great Ormond Street Hospital for Children, London, UK

P A Farndon, Clinical Genetics Unit, Birmingham Women's Hospital, Birmingham, UK

Ethical approval: This patient and family were part of a research study that was carried out with full ethical approval obtained from the ethics committee, Moorfields Eye Hospital, London, UK.

Correspondence to: Nicola K Ragge, MD, FRCPCH, FRCOphth, Department of Human Anatomy and Genetics, South Parks Road, Oxford OX1 3QX, UK; nicky.ragge@anat.ox.ac.uk

Accepted for publication 9 December 2004

\section{REFERENCES}

1 Ravine D, Ragge NK, Stephens D, et al. Dominant coloboma-microphthalmos syndrome associated with sensorineural hearing loss, hematuria, and cleft-lip/ palate. Am J Med Genet 1997;72:227-36.

2 Ragge NK, Ravine D, Wilkie AOM. Dominant inheritance of optic pits. Am J Ophthalmol 1998;125:124.

3 McLean CJ, Ragge NK, Jones RB, et al. The management of orbital cysts associated with congenital microphthalmos and anophthalmos. Br J Ophthalmol 2003;87:860-3.

4 Mclean JR, Boswell R, O'Donnell J. Cloning and molecular characterization of a metabolic gene with development functions in Drosophila. I. Analysis of the head function of Punch. Genetics 1990;126:1007-19.

5 Schimmenti LA, de la Cruz J, Lewis RA, et al. Novel mutation in sonic hedgehog in non-syndromic colobomatous microphthalmia. Am J Med Genet 2003; 116A:215-21.

6 Dubourg C, Lazaro L, Pasquier L, et al. Molecular screening of SHH, ZIC2, SIX3, and TGIF genes in patients with features of holoprosencephaly spectrum: mutation review and genotype-phenotype correlations. Hum Mutat 2004; $24: 43-51$

7 Leatherbarrow B, Kwartz J, Noble JL. Microphthalmos with cyst in monozygous twins. J Pediatr Ophthalmol Strabismus 1990;27:294-8.

8 Porges Y, Gershoni-Baruch R, Leibu R, et al. Hereditary microphthalmia with colobomatous cyst. Am J Ophthalmol 1992;114:30-4.

9 Lehman DM, Sponsel WE, Stratton RF, et al. Genetic mapping of a novel Xlinked recessive colobomatous microphthalmia. Am J Med Genet 2001;101:114-19.

10 Bar-Yosef U, Abuelaish I, Harel T, et al. CHX10 mutations cause nonsyndromic microphthalmia/anophthalmia in Arab and Jewish kindreds. Hum Genet 2004

11 Gorlin R, Goltz R. Multiple nevoid basal cell epithelioma, jaw cysts and bifid rib: a syndrome. N Engl J Med 1960;262:908.

12 Manners RM, Morris RJ, Francis PJ, et al. Microphthalmos in association with Gorlin's syndrome. Br J Ophthalmol 1996;80:378. 
13 Black GC, Mazerolle CJ, Wang Y, et al. Abnormalities of the vitreoretinal interface caused by dysregulated Hedgehog signaling during retinal development. Hum Mol Genet 2003; 12:3269-76.

14 Wicking C, Shanley S, Smyth I, et al. Most germ-line mutations in the nevoid basal cell carcinoma syndrome lead to a premature termination of the PATCHED protein, and no genotype-phenotype correlations are evident. Am J Hum Genet 1997;60:21-6.

15 Hahn H, Wicking C, Zaphiropoulous PG, et al. Mutations of the human homolog of Drosophila patched in the nevoid basal cell carcinoma syndrome. Cell 1996:85:841-51.

16 Villavicencio EH, Walterhouse DO, lannaccone PM. The sonic hedgehogpatched-gli pathway in human development and disease. Am J Hum Genet 2000;67:1047-54.

17 Belloni E, Muenke M, Roessler E, ef al. Identification of Sonic hedgehog as a candidate gene responsible for holoprosencephaly. Nat Genet 1996; 14:353-6.

18 Roessler E, Belloni E, Gaudenz K, et al. Mutations in the human Sonic hedgehog gene cause holoprosencephaly. Nat Genet 1996;14:357-60.

19 Roessler E, Belloni E, Gaudenz K, et al. Mutations in the C-terminal domain of Sonic Hedgehog cause holoprosencephaly. Hum Mol Genet 1997;6: 1847-53

20 Goodrich LV, Milenkovic L, Higgins KM, et al. Altered neural cell fates and medulloblastoma in mouse patched mutants. Science 1997;277:1109-13.

21 Milenkovic L, Goodrich LV, Higgins KM, et al. Mouse patched1 controls body size determination and limb patterning. Development 1999;126:4431-40.

22 Knudson AGJ. Mutation and Cancer: statistical study of retinoblastoma. Proc Natl Acad Sci 1971;68:820-3.
23 Levanat S, Gorlin RJ, Fallet S, et al. A two-hit model for developmental defects in Gorlin syndrome. Nat Genet 1996;12:85-7.

24 Bonifas JM, Bare JW, Kerschmann RL, et al. Parental origin of chromosome 9q22.3-q31 lost in basal cell carcinomas from basal cell nevus syndrome patients. Hum Mol Genet 1994;3:447-8.

25 Cowan R, Hoban P, Kelsey A, et al. The gene for the naevoid basal cell carcinoma syndrome acts as a tumour-suppressor gene in medulloblastoma. Br J Cancer 1997:76:141-5.

26 Pomeroy SL, Tamayo P, Gaasenbeek M, et al. Prediction of central nervous system embryonal tumour outcome based on gene expression. Nature 2002;415:436-42.

27 O'Malley S, Weitman D, Olding M, et al. Multiple neoplasms following craniospinal irradiation for medulloblastoma in a patient with nevoid basal cell carcinoma syndrome. Case report. J Neurosurg 1997;86:286-8.

28 Atahan IL, Yildiz F, Ozyar E, et al. Basal cell carcinomas developing in a case of medulloblastoma associated with Gorlin's syndrome. Pediatr Hematol Oncol 1998;15:187-91

29 Ratcliffe JF, Shanley S, Chenevix-Trench G. The prevalence of cervical and thoracic congenital skeletal abnormalities in basal cell naevus syndrome; a review of cervical and chest radiographs in 80 patients with BCNS. Br J Radiol 1995;68:596-9.

30 Evans DG, Farndon PA. Nevoid basal cell carcinoma syndrome. In: Washington U, ed. GeneReviews at gene tests: Medical Genetics Information Resource [database online]. Available at www.genetests.org. Seattle, 19972004 (updated October 2004) 